\title{
An intervention to increase consumption of bitter vegetables in late pregnancy reduces infants' disliking of bitter vegetables at weaning
}

\author{
J.E. Miller ${ }^{1}$, L. Milne ${ }^{1}$, J. Duncan ${ }^{1}$ and J.M. Wallace ${ }^{1}$ \\ ${ }^{1}$ Rowett Institute, University of Aberdeen, Aberdeen, UK
}

This abstract was presented as the Public Health Nutrition Theme highlight.

Children have an innate dislike for bitter foods such as green vegetables, however, repeated exposure to flavours can increase acceptance of them ${ }^{(1)}$. The maternal diet provides infants' first experiences of flavours in utero ${ }^{(1)}$ providing an opportunity to encourage healthful childhood dietary patterns via enhanced maternal fruit and vegetable $(\mathrm{F} \& \mathrm{~V})$ consumption - particularly bitter vegetables.

Women in late-pregnancy $(\mathrm{n}=55)$ ate soups with (treatment) or without (control) bitter vegetables for 24-days in addition to their usual diet. Habitual F\&V consumption was assessed by questionnaire at $\sim 30$ weeks gestation which yielded F\&V and bitter vegetable $(\max =300)$ frequency scores, and average portions per day consumed (portions/day). Milk-feeding scores for six-months postnatal were calculated from milk diaries $(0=$ exclusively formula-fed to $6=$ exclusively breastfed $)$. Infant taste-tests were videoed by the mothers in their own homes at the outset of weaning (mean 24.7 weeks). Potato puree was offered first until they were competent at eating, then taste-tests were conducted using apple, broccoli, and spinach purees. The first ten spoons were observed by four independent assessors to score negative facial expressions, negative/positive behaviours, and overall disliking (expressions and behaviours combined) using The Feeding Infants: Behaviour and Facial Expression Coding System ${ }^{(2)}$ (FIBFECS) - a validated method of assessing infants' wanting and liking of food. Mothers' perception of liking was recorded on a Likert-scale $(1=$ extremely disliked to $9=$ extremely liked).

Multiple regression identified the variables which predicted measures of infants' liking (FIBFECS measures and mothers' perception scores) of the purees. The model terms included maternal age, pre-pregnancy BMI, education, milk-feeding score, parity, weaning age, portions/day, intervention arm (control/treatment), bitter vegetable frequency score, and the interaction between intervention arm and bitter vegetable frequency score. For spinach, higher bitter vegetable frequency scores predicted more positive behaviours $(\beta 1.2 \mathrm{P}$ $=0.025)$, fewer negative behaviours $(\beta-0.4 \mathrm{P}=0.013)$ and expressions $(\beta-0.9 \mathrm{P}=0.002)$, lower overall disliking scores $(\beta-0.05 \mathrm{P}<0.001)$ and higher liking as perceived by the mothers $(\beta 0.1 \mathrm{P}<0.001)$. Treatment group similarly predicted positive $(\beta 45.2 \mathrm{P}=0.004)$ and negative $(\beta-13 \mathrm{P}=0.008)$ behaviours, overall disliking $(\beta-1.1 \mathrm{P}=0.001)$, and mothers' liking scores $(\beta 4.3 \mathrm{P}<0.001)$. Taking habitual bitter vegetable consumption into account (interaction-term), mother-infant dyads in the treatment group had higher scores for positive behaviours $(\beta-1.7 \mathrm{P}=0.014)$ and liking as perceived by the mothers $(\beta-0.1 \mathrm{P}=0.004)$, and lower scores for negative behaviours $(\beta 0.4 \mathrm{P}=0.037)$, expressions $(\beta 0.8 \mathrm{P}=0.025)$ and overall disliking $(\beta 0.05 \mathrm{P}<0.001)$. Treatment group predicted higher positive behaviour scores for broccoli $(\beta 31.1 \mathrm{P}=0.048)$, and higher negative expressions scores for apple $(\beta 12.1 \mathrm{P}=0.045)$.

Regular inclusion of bitter vegetables in the maternal diet during late pregnancy can increase infants' exposure to these flavours in utero and subsequent liking at weaning, and a dietary intervention to improve intakes in habitually low consumers has similar benefits. These early influences may in turn establish healthy dietary habits throughout the life-course.

\section{Acknowledgements}

Funded by the Scottish Government's Rural and Environment Science and Analytical Services Division

\section{References}

1. Ventura A \& Worobey J (2013) Curr Biol 23, 401-8.

2. Nekitsing C, Madrelle J, Barends C, et al. (2016) Food Qual Prefer 48, 228-237. 